\title{
MT1DP loaded by folate-modified liposomes sensitizes erastin-induced ferroptosis via regulating miR-365a-3p/NRF2 axis in non-small cell lung cancer cells
}

\author{
Chengcheng Gai ${ }^{1}$, Chuanliang Liư ${ }^{2}$ Xinghan $\mathrm{Wu}^{1}$, Mengyu $\mathrm{Yu}^{1}$, Jie Zheng ${ }^{1}$, Weifen Zhang ${ }^{3}$, Shijun $\mathrm{Lv}^{1}$ and \\ Wentong $\mathrm{Li}^{1}$
}

\begin{abstract}
Although ferroptosis has been recognized as a novel antitumoral treatment, high expression of nuclear factor erythroid 2-related factor 2 (NRF2) has been reported to be an antioxidant transcript factor that protects malignant cells from ferroptosis. Previous findings indicated that metallothionein 1D pseudogene (MT1DP), a long noncoding RNA (InCRNA), functioned to aggravate oxidative stress by repressing antioxidation. Here we aimed at assessing whether MTIDP could regulate erastin-induced ferroptosis on non-small cell lung cancer (NSCLC) and elucidating the mechanism. We found that ectopic expression of MT1DP sensitized A549 and H1299 cells to erastin-induced ferroptosis through downregulation of NRF2; in addition, ectopic MT1DP upregulated malondialdehyde (MDA) and reactive oxygen species (ROS) levels, increased intracellular ferrous iron concentration, and reduced glutathione (GSH) levels in cancer cells exposed to erastin, whereas downregulation of MT1DP showed the opposite effect. RNA pulldown assay and dual-luciferase reporter assay confirmed that MT1DP modulated the expression of NRF2 via stabilizing miR-365a-3p. As low solubility of erastin limits its efficient application, we further prepared folate (FA)modified liposome (FA-LP) nanoparticles for targeted co-delivery of erastin and MTIDP to enhance the bioavailability and the efficiency of the drug/gene combination. Erastin/MT1DP@FA-LPs (E/M@FA-LPs) sensitized erastin-induced ferroptosis with decreased cellular GSH levels and elevated lipid ROS. In vivo analysis showed that E/M@FA-LPs had a favorable therapeutic effect on lung cancer xenografts. In short, our findings identify a novel strategy to elevate erastin-induced ferroptosis in NSCLCs acting through the MT1DP/miR-365a-3p/NRF2 axis.
\end{abstract}

\section{Introduction}

Ferroptosis, which is a novel subtype of cell death different from other types of cell death both morphologically and biochemically ${ }^{1,2}$, is characterized by depletion of glutathione $(\mathrm{GSH})$ and overgeneration of lipid reactive

\footnotetext{
Correspondence: Shijun Lv (sjlu@wfmc.edu.cn) or

Wentong Li (liwentong11@163.com)

'Department of Pathology, Weifang Medical University, Weifang, Shandong

Province 261014, China

${ }^{2}$ The Second Department of Health Care, Weifang People's Hospital, Weifang, Shandong Province 261041, China

Full list of author information is available at the end of the article

Edited by F. Pentimalli
}

oxygen species (ROS) depending upon intracellular iron ions ${ }^{1,3}$.

Erastin has been identified as a prototype of recently discovered Ras-selective ferroptotic compounds ${ }^{4}$; however, previous studies confirmed that a variety of tumor cells were insensitive to erastin ${ }^{5}$. nuclear factor erythroid 2-related factor 2 (NRF2) has been reported to be an important transcription factor that protects malignant cells from oxidative stress, chemotherapeutic agents, and facilitates cancer progression ${ }^{6,7}$. Suppression of NRF2 contributed to an increased oxidative stress level and accelerated ferroptosis ${ }^{8,9}$.

\section{(c) The Author(s) 2020}

(c) (i) Open Access This article is licensed under a Creative Commons Attribution 4.0 International License, which permits use, sharing, adaptation, distribution and reproduction cc. in any medium or format, as long as you give appropriate credit to the original author(s) and the source, provide a link to the Creative Commons license, and indicate if changes were made. The images or other third party material in this article are included in the article's Creative Commons license, unless indicated otherwise in a credit line to the material. If material is not included in the article's Creative Commons license and your intended use is not permitted by statutory regulation or exceeds the permitted use, you will need to obtain permission directly from the copyright holder. To view a copy of this license, visit http://creativecommons.org/licenses/by/4.0/. 
A growing number of evidences has suggested that some long noncoding RNAs (lncRNAs) are involved in cancer initiation and progression. Recently, IncRNAs have been found to act as part of the cellular antioxidant system that orchestrates signaling pathways to fine-tune cell survival and death in response to external stresses ${ }^{10,11}$. Previous findings indicated that an IncRNA, metallothionein 1D pseudogene $(M T 1 D P)$, functioned to aggravate oxidative stress by repressing NRF2-mediated antioxidation ${ }^{12,13}$.

Here we confirmed that MT1DP attenuated expression of NRF2 and increased sensitivity of NRF2-overexpressed non-small cell lung cancer (NSCLC) cells to erastininduced ferroptosis via stabilizing miR-365a-3p. We demonstrated that the cell viability of NSCLC cell lines was significantly restrained by erastin and exogenous $M T 1 D P$ accompanied with augmented lipid peroxidation. We further developed a nano-delivery system based on folate-modified liposomes (FA-LP) to co-delivery MT1DP and erastin(E/M@FA-LPs). In vitro and in vivo experiments indicated that E/M@FA-LPs displayed strong killing effects on tumor cells.

\section{Material and methods Cell culture}

KEAP1 mutant NSCLC cell lines (A549) and KEAP1 non-mutant NSCLC cell lines (H1299) were acquired from American Type Culture Collection (Manassas, VA, USA). A549, H1299 were cultured in RPMI-1640 supplemented with $10 \%$ fetal bovine serum (FBS; Invitrogen, CA, USA). All cell lines were maintained at $37^{\circ} \mathrm{C}$ and $5 \%$ $\mathrm{CO}_{2}$ in a humid environment.

\section{Tissue samples}

A total of 64 cases of human NSCLC tissue samples (32 cases with mutant KEAP1, 32 cases with non-mutant KEAP1) from November 2013 to October 2017 were pathologically diagnosed at the Affiliated Hospital of Weifang Medical University. Prior informed consent for the use of tissue samples was obtained from all patients and the study was approved by the Ethics Committees of the Weifang Medical University.

\section{Bioinformatics analysis}

Ferroptosis-inducer data for differential genetic analysis were obtained from NCBI publicly available genomics database, Gene Expression Omnibus (GEO) DataSets (GSE94550, GSE137952, and GSE122985). The R software package was used to process and identify the differential expressed genes.

\section{Preparation of LPs for co-loading of erastin and MT1DP}

Precisely weighed $120 \mathrm{mg}$ of $\mathrm{L}-\alpha$-phosphatidylcholine (Solarbio, Beijing, China), $40 \mathrm{mg}$ of cholesterol (Solarbio), $20 \mathrm{mg}$ of DSPE-PEG-FA (Shanghai Ponsure Biotechnology,
China, Shanghai), and $3 \mathrm{mg}$ of erastin (Sigma-Aldrich, MO, USA) were fully dissolved in $5 \mathrm{~mL}$ chloroform and mixed with $3 \mathrm{~mL}$ of $1 \mathrm{mg} / \mathrm{mL}$ erastin chloroform solution. Chloroform was then removed using a rotary evaporator to form a dry-lipid film at $35^{\circ} \mathrm{C}$ under uniform speed. MT1DP (300 nM) dissolved in phosphate-buffered saline (PBS) $(\mathrm{pH} 7.4,5 \mathrm{~mL})$ was added to the lipid film and hydrated for $60 \mathrm{~min}$. After the hydration was completed, the cells were sonicated with an ultrasonic cell disruptor and passed through a polyethersulfone membrane with a membrane pore diameter of 450 and $220 \mathrm{~nm}$ to decrease the particle size.

\section{Loading capacity, encapsulation efficiency, and drug- release studies}

The E/M@FA-LPs suspension was filtered through a $0.22 \mu \mathrm{m}$ microfilter membrane, the quantity of erastin in the supernatant was analyzed using the reverse-phase high-performance liquid chromatography (HPLC) analysis method. encapsulation efficiency (EE \%) and loading capacity (LC \%) were calculated using equations as previously described ${ }^{14}$. The quantity of erastin was measured using the Shimadzu Prominence HPLC system and C18 analytic column (Luna C18(2) $25 \mathrm{~cm} \times 4.6 \mathrm{~cm}, 5 \mathrm{~mm}$, Phenomenex, Inc., Torrance, CA). The ultraviolet absorbance was measured at a wavelength of $275 \mathrm{~nm}$ and their cumulative release was calculated.

\section{Plasmid construction and cell transfection}

MiR-365 mimic (5'-UAAUGCCCCUAAAAAUCCUU AU- $3^{\prime}$ ) and miR-365 inhibitor (5'-AUAAGGACCCCCA GGGGCAUUA-3') were chemically synthesized by Sangon (Shanghai, China). MT1DP was subcloned into pEGFP-N1 vector as previously described ${ }^{15}$. MT1DP short hairpin RNA (shRNA) and NRF2 shRNA sequences were ligated into the pGPU6/GFP/Neo vector to construct siMT1DP and siNRF2 plasmids. pcDNA3-EGFPC4-NRF2 plasmid $^{16}$ was acquired from Addgene (Catalog: 21549; Cambridge, MA, USA). The overexpression plasmids and interference plasmids were extracted by QIAGEN Plasmid Extraction Kit. The mixture containing RNA-overexpressing or -interfering plasmid was added to Lipofectamine 2000 (Invitrogen, Carlsbad, CA, USA) and incubated in NSCLC cells.

\section{Cell viability assay}

Cells $\left(1 \times 10^{3} /\right.$ well $)$ were seeded in a 96-well plate and treated with or without erastin $(10 \mu \mathrm{M} / \mathrm{mL})$ for $48 \mathrm{~h}$. MTT (3-(4,5-dimethylthiazol-2-yl)-2,5-diphenyltetrazolium bromide, $5 \mathrm{mg} / \mathrm{mL})$ solution $(10 \mu \mathrm{L} /$ well $)$ was added into the medium and incubated for $4 \mathrm{~h}$; MTT solution was discarded and $100 \mu \mathrm{L}$ dimethyl sulfoxide was added. The absorbance was measured at $490 \mathrm{~nm}$ by SpectraMax M5 plate reader (Molecular Devices, Sunnyvale, CA, USA). 


\section{EdU proliferation assay}

To measure cell proliferation, 5-ethynyl-2'-deoxyuridine (EdU) proliferation assay was performed. Cells were plated in 24-well plates at a density of $5 \times 10^{4}$ cells/well. Twenty-four hours later, cells were treated with $10 \mu \mathrm{M}$ EdU (RiboBio, Guangzhou, China) and fixed with 4\% paraformaldehyde. The sections were imaged using a fluorescence microscope and the number of proliferating cells was averaged to calculate the labeling index.

\section{Wound-healing and transwell invasion assay}

Cells/well $\left(1 \times 10^{5}\right)$ were plated into a six-well plate and incubated for $24 \mathrm{~h}$ and a straight line was scratched across the surface of cells. After incubation for $24 \mathrm{~h}$, migration distance was calculated. Transwell invasion assay was performed using Millipore transwell chambers $(8 \mu \mathrm{m}$ pore size; Millipore, Billerica, MA, USA). A549 cell $\left(2 \times 10^{4}\right.$ cells/well) transfected with MT1DP were seeded in the top chamber in $100 \mu \mathrm{L}$ serum-free medium. The lower chambers were filled with $600 \mu \mathrm{L}$ complete medium with $10 \%$ FBS. After $24 \mathrm{~h}$ incubation, $0.1 \%$ crystal violet dye was used to stain cells. The images were analyzed by $\mathrm{NIH}$ ImageJ software (National Institutes of Health, Bethesda, MD, USA).

\section{ROS assay and lipid peroxidation assessment, and} measurement of intracellular GSH

Intracellular ROS was measured using dichlorofluorescein diacetate fluorescent probe detection kit according to the manufacturer's instruction (Thermo Fisher Scientific, Waltham, MA, USA), results were monitored with fluorescence microscope or SpectraMax M5 plate reader. Malondialdehyde (MDA) assay kit was used to assess lipid peroxidation according to the manufacturer's instruction; for the measurement of intracellular GSH, MT1DP overexpression cells were treated with or without erastin for $24 \mathrm{~h}$, cells were sonicated, and the supernatant was used to detect intracellular GSH by GSH assay kit (Beyotime Biotechnology, Nanjing, China). GSH content was expressed as a ratio to the absorbance, which was measured at $412 \mathrm{~nm}$.

\section{Ferrous iron assay}

Cells were plated at $1 \times 10^{5}$ cells/well in a six-well plate; $24 \mathrm{~h}$ later, they were treated with erastin and/or NRF2 inhibitor. The intracellular ferrous iron level was assessed with an iron colorimetric assay kit (Abcam, Cambridge, UK) according to the manufacturer's instruction.

\section{Flow cytometry}

Cells/well $\left(1 \times 10^{5}\right.$; A549 or H1299 cells) were plated in a six-well plate; $24 \mathrm{~h}$ later, $M T 1 D P$-overexpressing cells were treated or not with erastin. Cells were suspended in $100 \mu \mathrm{L}$ binding buffer and stained with Annexin V-fluorescein isothiocyanate (FITC) and 7-Aminoactinomycin (Annexin V-FITC Kit; Beckman Coulter, Marseille, France) according to the manufacturer's instruction. Cell ferroptosis was analyzed using flow cytometry (FCM; FACSCalibur, BD Biosciences, Franklin Lakes, NJ, USA).

\section{Transmission electron microscope assay}

For analyzing the morphology of mitochondria, $2 \times 10^{5}$ cells/well were seeded in 6-well plate and exposed to erastin for $12 \mathrm{~h}$. After that, cells were collected, washed three times with PBS, and fixed with $2.5 \%$ glutaraldehyde. Samples were then pretreated according to standard procedures including staining, dehydration, embedding, and slicing to obtain ultra-thin sections. During the analysis, images were acquired using a HITACHIH-7000 transmission electron microscope (TEM; Hitachi, Tokyo, Japan).

\section{Mitochondrial membrane potential $(\Delta \psi \mathrm{M})$ assay}

Fluorescent dye 5, 5', 6, 6'-tetrachloro-1,1',3,3'-tetraethyl benzimidazolyl-carbocyanineio-dide (JC-1, Beyotime Biotech, Nanjing, China) was used to monitor $\Delta \psi \mathrm{M}$. Cells were seeded in a six-well plate at $3 \times 10^{5} \mathrm{cell} /$ well . Loss of $\Delta \psi \mathrm{M}$ was determined by decrease in JC-1 aggregates (red) and increase in JC-1 monomers (green). Cells were visualized by laser scanning confocal microscope (Leica TCS SP8, Solms, Germany). The excitation wavelength is $488 \mathrm{~nm}$ and the emission wavelengths of the JC-1 monomeric form and JC-1 aggregate form were 529 and $590 \mathrm{~nm}$, respectively. The images were analyzed by $\mathrm{NIH}$ ImageJ software.

\section{Dual-luciferase reporter assay}

The $3^{\prime}$-untranslated region (UTR) fragment of NRF2 mRNA containing the potential binding site of miR-365a$3 p$ was inserted into a pMIR-REPORTTM luciferase reporter vector (Ambion, Austin, TX, USA) and the resulting plasmid was named NRF2-3'-UTR-WT. Another vector containing a mutant NRF2-3'-UTR at the binding site of miR-365a-3p was constructed and named NRF2-3'-UTR-MUT. Luciferase activity was measured in the form of chemiluminescence using the dual-luciferase reporter assay system (Promega, Madison, MI, USA) according the manufacturer's instruction.

\section{RNA pulldown assay}

A biotinylated DNA probe, similar to the sequence of miRNA-365, was synthesized and dissolved in $500 \mathrm{~mL}$ wash/binding buffer $(0.5 \mathrm{M} \mathrm{NaCl}, 20 \mathrm{mM}$ Tris- $\mathrm{HCl}$, and $1 \mathrm{mM}$ EDTA). The probe was incubated within cell lysates from A549 and H1299 cells $\left(1 \times 10^{7}\right)$, then streptavidin-coated magnetic beads (Sigma) were added for $2 \mathrm{~h}$ at $25^{\circ} \mathrm{C}$. The $M T 1 D P$ present in the pulldown complex was detected by quantitative reverse transcriptase PCR analysis. 


\section{Western blot analysis}

Cell were lysed using NP-40 lysis buffer (Roche, Basel, Switzerland) and the extracted proteins were separated by SDS-polyacrylamide gel electrophoresis and transferred onto polyvinylidene difluoride transfer membrane (Merck Millipore, Billerica, USA). The membrane was incubated with the appropriate anti-NRF2 and anti-GAPDH antibodies (Abcam, Cambridge, UK) overnight at $4{ }^{\circ} \mathrm{C}$. The blots were developed with a peroxidase-conjugated secondary antibody and the proteins were visualized using by ECL Plus Detection Reagent (Merck Millipore, Billerica, USA). The gray-scale value was assessed by Gel-Pro analyzer.

\section{Quantitative real-time PCR}

Total RNA was isolated using Trizol (Invitrogen, CA, USA) and first-strand complementary DNA was synthesized using the Reverse Transcription System Kit according to the manufacturer's instructions (Takara Bio, Shiga, Japan). Complementary DNA from various cell samples was amplified with specific primers. NRF2: $5^{\prime}-\mathrm{C}$ ACATCCAGTCAGAAACCAGTGG-3' and $5^{\prime}$-GGAAT GTCTGCGCCAAAAGCTG-3'; MT1DP: 5'-TCAAGG CCAAAGGTGGCTCCTGCAC-3' ${ }^{\prime}$, and $5^{\prime}$-GCACGGCA GCTGCACTTCACCAATG-3'; miR-365a-3p: 5'-TGCG GTAATGCCCCTAAAAA- ${ }^{\prime}$ ' and $5^{\prime}$-TGCAAGAGCAAT AAGGATT-3'; U6: $5^{\prime}$-CTCGCTTCGGCAGCACA- $3^{\prime}$ and $5^{\prime}$-AACGCTTCACGAATTTGCGT-3'; GAPDH: $5^{\prime}$-CAATGACCCCTTCATTGACC- $3^{\prime}$ and $5^{\prime}$-GACAAG CTTCCCGTTCTCAG-3'.

\section{In vivo study}

All animal experiments were approved by the Ethics Committee of Weifang Medical University. A total of $6 \times$ $10^{6}$ A549 cells were subcutaneously injected into the right flank of the athymic BALB/c nude mice (aged 4 weeks, weight 12-16 g; Vital River, Beijing, China). Once tumors reached about $80 \mathrm{~mm}^{3}$, the mice were randomly divided into four groups. Mice were treated with $50 \mu \mathrm{M} / \mathrm{kg}$ erastin by intraperitoneal injection every 2 days for eight times. Tumor size was measured and calculated with the following formula $0.5 \times$ length $\times$ width $^{2}$. The tumor tissues, hearts, lungs, spleens, kidneys, and liver were fixed with $4 \%$ paraformaldehyde for immunohistochemistry and hematoxylin and eosin staining.

\section{Immunohistochemistry}

Immunohistochemistry for NRF2 was performed on paraffin-embedded xenograft tumor tissue sections. The slides were incubated with primary antibody targeting NRF2 ( $1: 200$; Abcam, Cambridge, UK) overnight at $4{ }^{\circ} \mathrm{C}$. Slides were incubated with horseradish peroxidase-conjugated anti-rabbit secondary antibody (Protein Tech, IL, USA) for $1 \mathrm{~h}$, then the slides were developed with diaminobenzidine. The images were analyzed by NIH imageJ software.

\section{Statistical analysis}

All data were shown as mean \pm SD and analyzed with GraphPad Prism 7 software (La Jolla, CA, USA). Statistical analysis was analyzed by independent $t$-test or one-way analysis of variance test. A $P$-value $<0.05$ was considered statistically significant.

\section{Results \\ MT1DP sensitizes erastin-induced ferroptosis via repressing of NRF2 in NSCLC}

To identify candidate genes that are associated with ferroptosis sensitivity, we performed a bioinformatics analysis from the GEO database (i.e., GSE94550, GSE137952, and GSE122985). Briefly, we screened the data in which clinical drugs (e.g., sulfasalazine, sorafenib, and artesunate) were used to induce ferroptosis, these data displayed that MT1DP was one of the significantly downregulated genes in the ferroptosis-insensitive cell lines (Fig. 1a). Our previous studies have demonstrated that A549 and H1299 cells are insensitive to ferroptosis induced by erastin ${ }^{17}$, so we tested the effect of erastin on MT1DP expression. Our results found that MT1DP expression in NSCLC cells treated with erastin decreased (Fig. 1b). Silencing of NRF2 inhibited cell viability, whereas exogenous NRF2 increased cell viability in both A549 and H1299 cell lines (Fig. 1c, d and Supplementary Fig. S1A, B). A negative correlation between the MT1DP expression and NRF2 expression was identified (Fig. 1e). Knockdown or overexpression of NRF2 had no significant effects on MT1DP expression (Supplementary Fig. S1C, D). NRF2 expression was downregulated when MT1DP was overexpressed in A549 and H1299 cells (Fig. 1f, g and Supplementary Fig. S2A). On the contrary, MT1DP silencing elevated the expression of NRF2 (Fig. 1h, i and Supplementary Fig. S2B). A549 and H1299 overexpressing $M T 1 D P$ were more sensitive to erastin compared to the control cells as confirmed by cell viability assay (Fig. 1j); at the same time, ROS was excessively generated (Fig. 1k). The cytotoxicity induced by MT1DP in combination with erastin could be relieved in the presence of ferrostatin-1 (Fig. 11), as shown by MTT. Overexpression of exogenous NRF2 rescued $M T 1 D P$-mediated sensitivity to erastin (Fig. $1 \mathrm{~m}$ ). These results indicated that MT1DP promoted the occurrence of cellular oxidative stress.

\section{MT1DP represses NRF2 via stabilizing miR-365a-3p}

RAID v2.0 was used to identify molecules that interacted with $M T 1 D P$. Indeed, we observed that hsa-miR$365 a-3 p$ achieved one of the highest confidence scores among all types of interactors (Fig. 2a); MT1DP and NRF2-3'-UTR share a consensus binding site on miR$365 a-3 p$ as revealed through bioinformatics prediction (Fig. 2b). We searched for partner molecules that could bind both MTIDP and miR-365a-3p, and performed an 


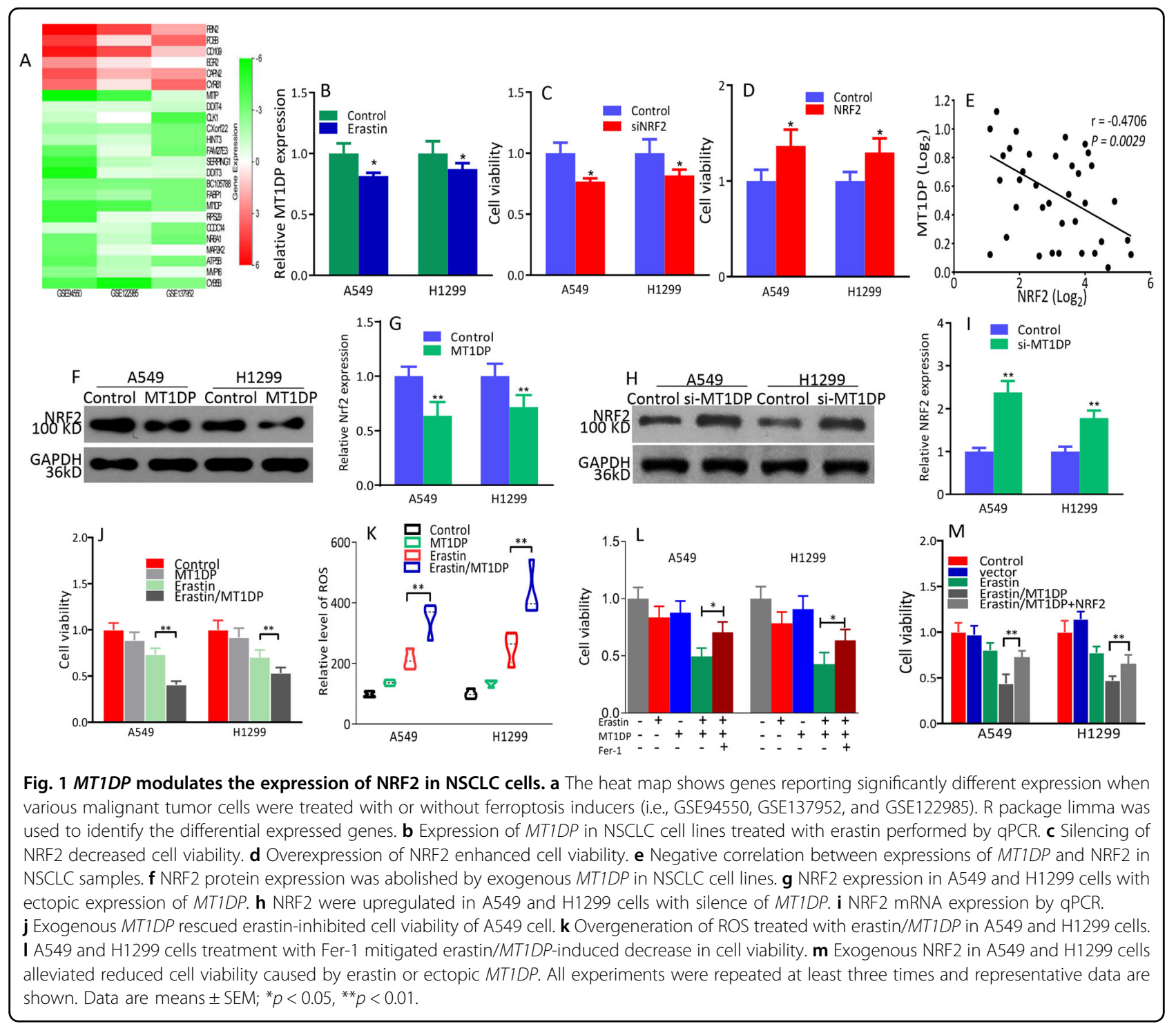

RNA pulldown experiment using biotin-labeled MT1DP and antisense-MT1DP as control. As shown in Fig. 2c, miR-365a-3p was more highly enriched in MT1DP pulldown cell lysate compared with the control, as shown by quantitative PCR analysis. MiR-365a-3p was enhanced and NRF2 was suppressed in MT1DP-overexpressing A549 and H1299 cells relative to control cells (Fig. 2d). MiR-365a-3p inhibitor abrogated the downregulation of NRF2 observed upon MT1DP overexpression (Fig. 2e). Dual-luciferase assay was carried out to validate NRF2 as a target of miR-365a-3p; results revealed that miR-365a-3p could suppress the luciferase activity of NRF2-3'-UTR-WT but it had less effect on NRF2-3'-UTR-MUT (Fig. 2f). MiR-365a-3p mimic reduced mRNA and protein expression of NRF2 in A549 and H1299 cells (Fig. 2g, h).

\section{Characterization of the erastin/MT1DP co-loaded FA-LPs}

We loaded erastin and MT1DP into FA-labeled liposomes to prepare E/M@FA-LPs (Fig. 3a). The diameter of FA-LPs was $139.7 \mathrm{~nm}$, as shown by dynamic light scattering (DLS) analysis (Supplementary Fig. S3A). The characterization of E/M@FA-LPs by DLS and TEM, showed that E/M@FA-LPs had a size distribution of $\sim 174 \mathrm{~nm}$ by DLS, polymer dispersity index of 0.307 , and zeta potential was $-24 \mathrm{mV}$ (Fig. 3b). As shown in the TEM images, E/M@FA-LPs were confirmed to be of spherical shape and uniform size, and had a particle diameter of about $154 \mathrm{~nm}$ (Fig. 3c). The LC and EE of E/ M@FA-LPs measured using HPLC are $18.98 \pm 2.24 \%$ and $48.87 \pm 4.83 \%$, respectively. As shown in Fig. $3 \mathrm{~d}$, the drugrelease profile of erastin reached $95 \%$ within the first $8 \mathrm{~h}$; release profile of E/M@FA-LPs had shown initial burst 

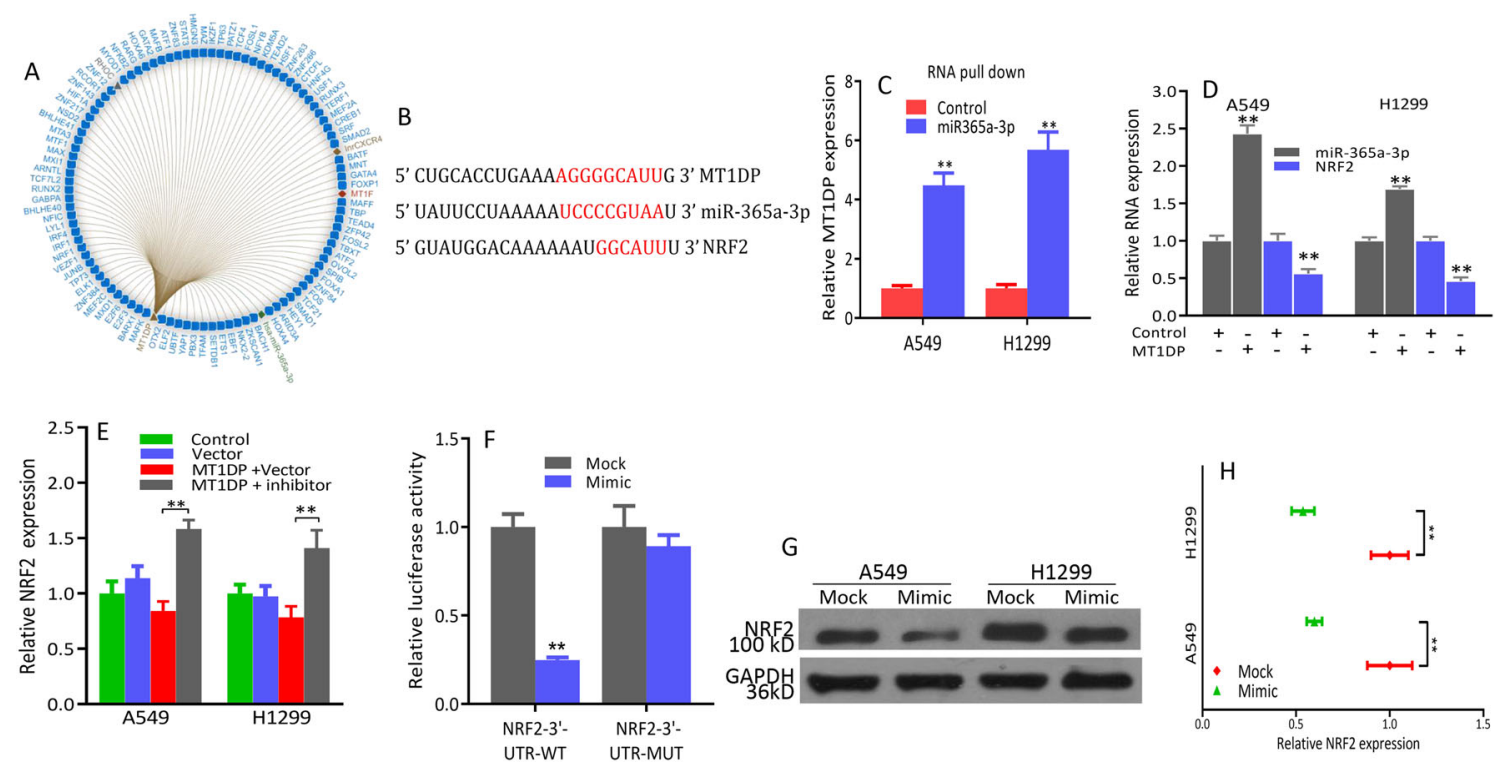

Fig. 2 MT1DP represses NRF2 via stabilizing miR-365a-3p. a The interaction network showed that miR-365a-3p was correlated with MT1DP significantly. $\mathbf{b}$ The binding site of miR-365a-3p within MT1DP and 3'-UTR of NRF2. $\mathbf{c}$ Interaction between miR-365a-3p and MT1DP was confirmed by RNA pulldown assay. d MT1DP overexpression elevated miR-365a-3p and reduced NRF2. e MT1DP-mediated NRF2 downregulation was abrogated by miR-365a-3p inhibitor. f NRF2 was identified as a target of miR-365a-3p by dual-luciferase reporter assay. $\mathbf{g}$ miR-365a-3p mimic decreased NRF2 expression. $\mathbf{h}$ miR-365a-3p mimic decreased NRF2 mRNA. All experiments were repeated at least three times and representative data are shown. Data are means \pm SEM; ${ }^{* *} p<0.01$.
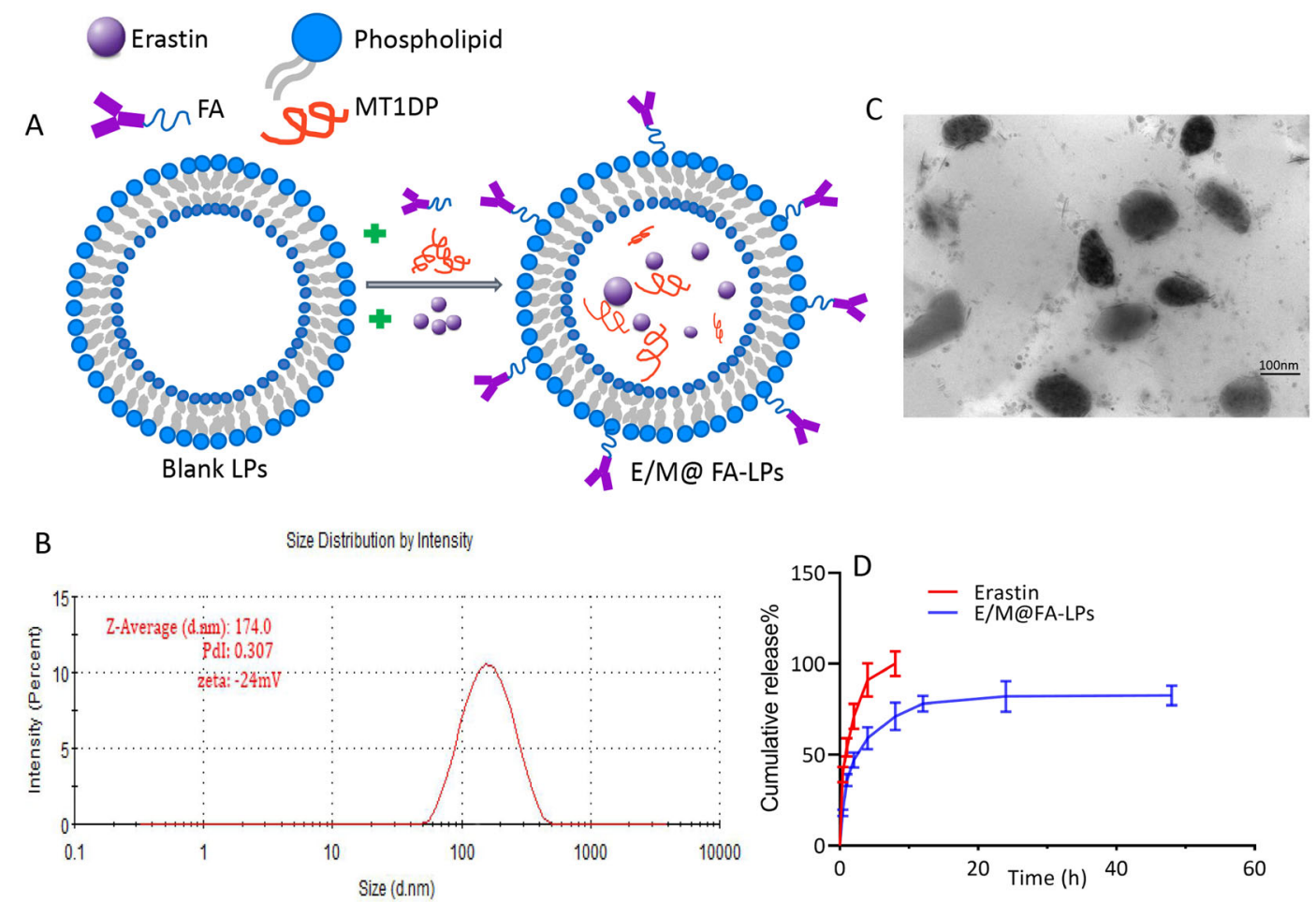

Fig. 3 Characterization of E/M@FA-LPs. a Schematic diagram of E/M@FA-LPs preparation. b The diameter of E/M@FA-LPs performed by DLS is about $174 \mathrm{~nm}$ and the zeta potential value was $-24 \mathrm{mV}$. c TEM images of E/M@FA-LPs. The micrographs show spherical particles for the E/M@FA-LPS, with the size of $154 \mathrm{~nm}$. Scale bar represents $100 \mathrm{~nm}$. d Cumulative erastin release from E/M@FA-LPs. All experiments were repeated at least three times and representative data are shown. Data are means \pm SEM. 


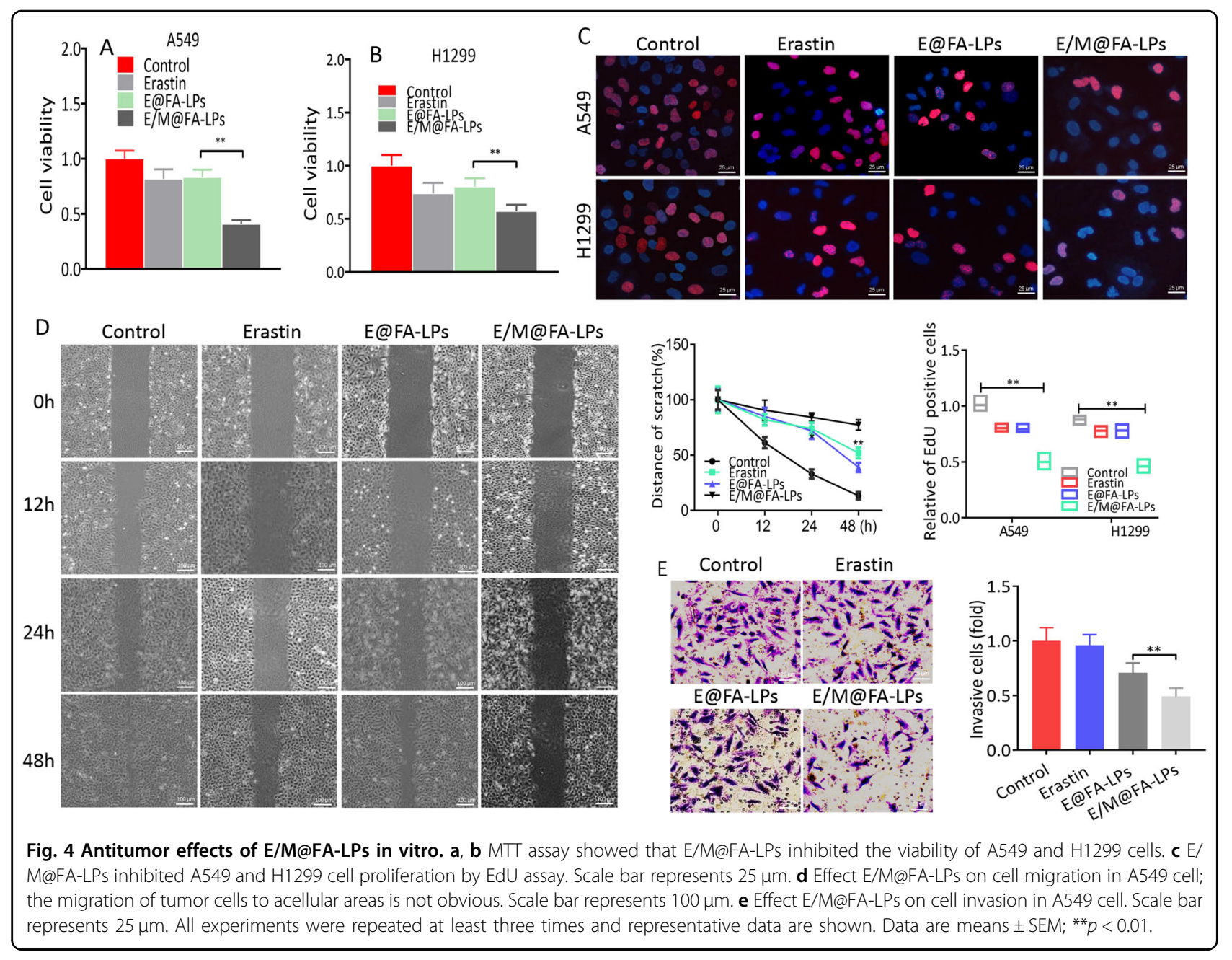

release in the early $2 \mathrm{~h}$ followed by a sustained release. Similarly, MT1DP is also released from E/M@FA-LPs slowly (Supplementary Fig. S3B).

\section{E/M@FA-LPs suppress cell malignance}

MTT results indicated that E/M@FA-LPs remarkably inhibited cell viability in A549 or H1299 cells compared to E@FA-LPs (Fig. 4a, b). EdU assay was performed to explore the biological effect of E/M@FA-LPs on cell proliferation. As expected, A549 or H1299 cell proliferation was inhibited by E/M@FA-LPs (Fig. 4c). The woundhealing assays demonstrated that the cell migration ability was suppressed significantly upon treatment with E/ M@FA-LPs (Fig. 4d). In addition, transwell invasion assay suggested that E/M@FA-LPs significantly reduced the number of invading cells (Fig. 4e).

E/M@FA-LPs sensitizes erastin-induced ferroptosis in vitro

FCM analysis confirmed that E/M@FA-LPs induced higher levels of ferroptosis compared to those induced by erastin or E@FA-LPs alone (Fig. 5a). Meanwhile, results of
TEM brought to light that E/M@FA-LPs contributed to the characteristic changes of ferroptosis on mitochondria with significantly decreased cristae, shrunken mitochondria, and increased membrane density in A549 and H1299 cells (Fig. 5b). Redox-active iron has been implicated as a central player in ferroptosis, so we assessed the iron levels in NSCLC cells treated with E/M@FA-LPs. Intracellular ferrous iron was upregulated significantly in E/M@FALPs-treated cells (Fig. 5c).

\section{E/M@FA-LPs represses NRF2 levels to enhance oxidative stress}

ROS, MDA, and GSH were detected to explore the effect of E/M@FA-LPs-induced ferroptosis. ROS and MDA generation were significantly increased in E/ M@FA-LPs compared to erastin in A549 and H1299 cells (Fig. 6a, b); in addition, GSH levels were significantly declined by E/M@FA-LPs compared to erastin (Fig. 6c). Previous results suggest that voltage-dependent anion channels play a critical role in the formation of mitochondrial ROS and own potential therapeutic value 

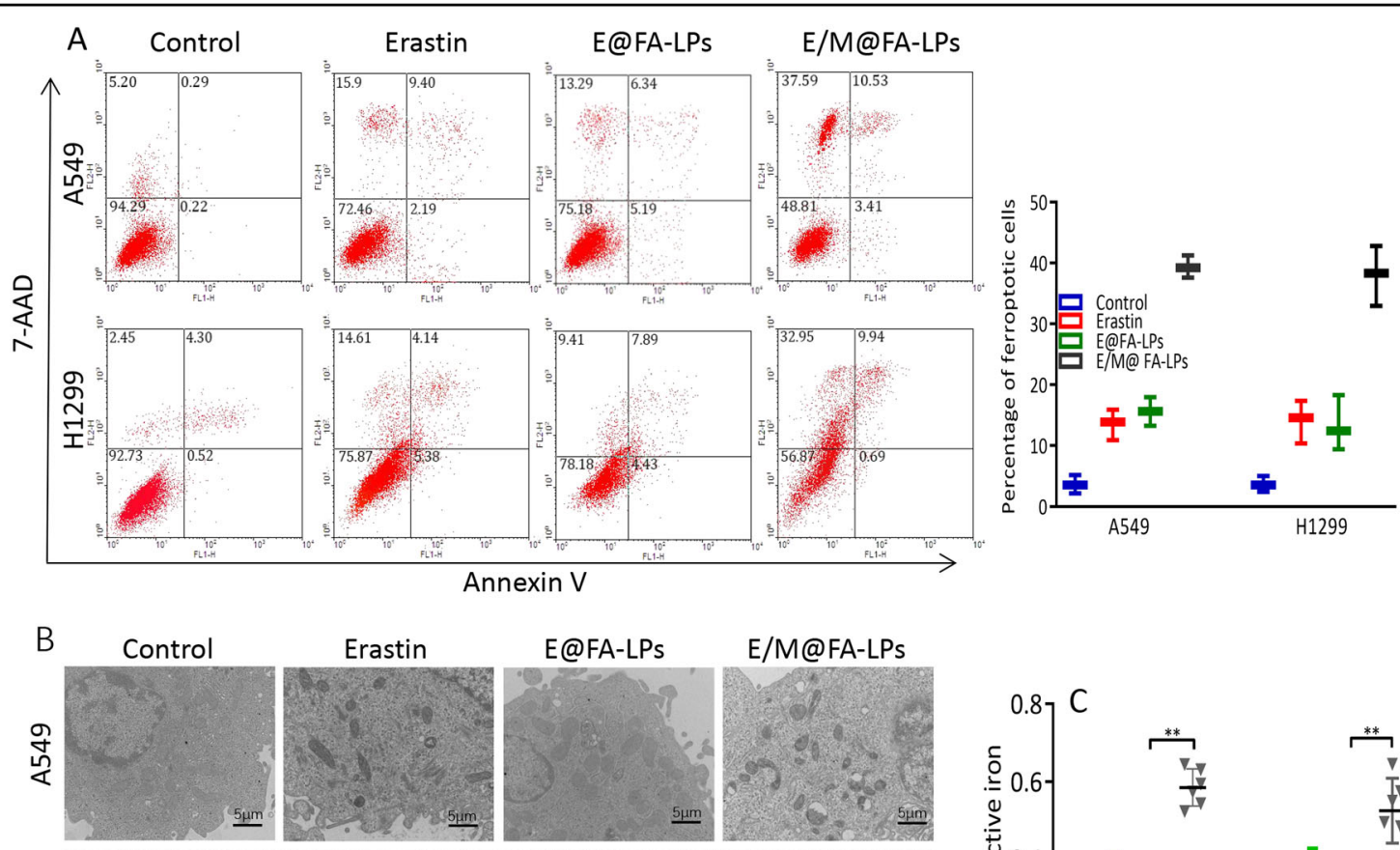

E@FA-LPs

E/M@FA-LPs
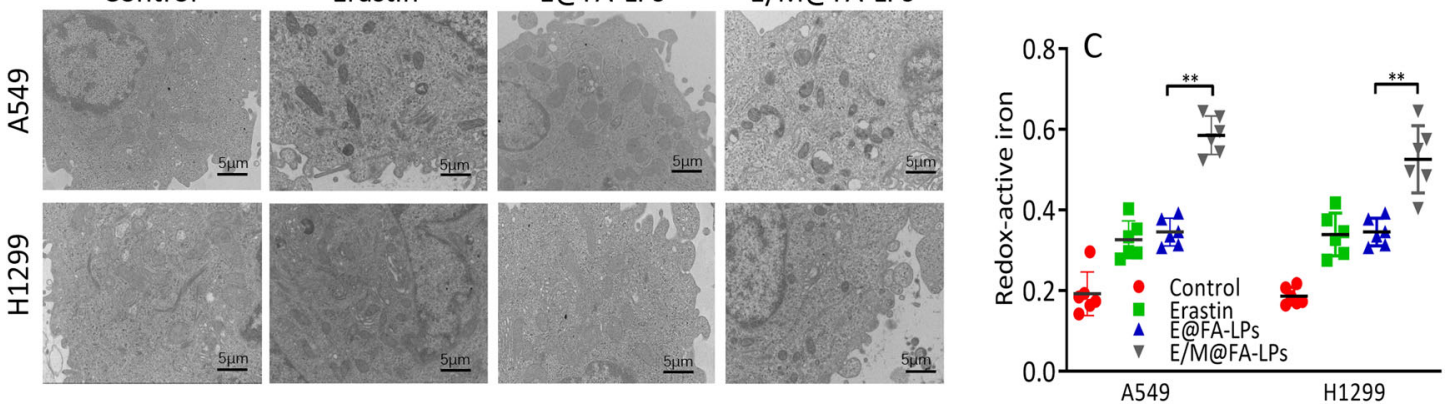

Fig. 5 E/M@FA-LPs led to ferroptosis. a E/M@FA-LPs contributed to ferroptosis in A549 cell as confirmed by flow cytometry. b The A549 and H1299 cells treated with E/M@FA-LPs showed shrunken mitochondria with increased membrane density. Scale bar represents $5 \mu$ m. c E/M@FA-LPs increased intracellular ferrous iron in A549 and H1299 cells. All experiments were repeated at least three times and representative data are shown. Data are means \pm SEM; ${ }^{* *} p<0.01$.

against glutamate-mediated oxidative toxicity. Here we found that A549 cell treated with E/M@FA-LPs showed higher accumulation of JC-1 monomers and lower accumulation of JC-1 aggregates (Fig. 6d).

\section{In vivo antitumor effect of E/M@FA-LPs}

E/M@FA-LPs effectively reduced the size of tumors formed by A549 cells compared to E@FA-LP-treated group (Fig. 7a). No significant loss in average body weight occurred (data not shown). The weight of xenograft tumors treated with E/M@FA-LPs was much lighter compared to those from the E@FA-LP-treated group (Fig. 7b). As illustrated in Fig. 7c, more cell death was shown after being treated with E/M@FA-LPs group, whereas only moderate level of cell death was identified in the mice treated with erastin. Tumors were further analyzed by immunohistochemistry, similar to in vitro results, NRF2 expression was decreased in the E/M@FA-LP group (Fig. 7c). MiR-365a-3p expression was augmented in E/M@FA-LP group (Fig. 7d). In addition, E/M@FALPs also resulted in more overproduction of MDA (Fig. 7e).

\section{Discussion}

Erastin selectively kills NSCLC cells by inducing ROS accumulation and iron-dependent ferroptosis ${ }^{2}$; unfortunately, its low solubility and metabolic liability preclude its use ${ }^{4}$. Meanwhile, several studies have demonstrated that cancer cells with elevated NRF2 are insensitive to erastin-induced ferroptosis ${ }^{18}$. Therefore, inhibition of the NRF2 signaling pathway could be exploited to induce ferroptosis of NSCLC cells ${ }^{8,19}$.

In this study, we found that downregulation of NRF2 contributed to decrease significantly the viability of A549 and H1299 cells treated with erastin; conversely, overexpression of NRF2 rescued erastin-induced ferroptosis. This might be due to the fact that erastin induces ferroptosis by inhibiting $\mathrm{xCT}$ and NRF2 is known to upregulate $\mathrm{xCT}$. Previous findings revealed an important role of MT1DP in calibrating the cellular machinery to switch the cellular defense to cytotoxicity through crosslinking with $M T 1 H^{12}$, which plays a crucial role in redox balance $^{20,21}$. Our data clearly demonstrated that exogenous MT1DP augmented the effect of erastin on A549 and H1299 cells with excessive production of peroxides. To 


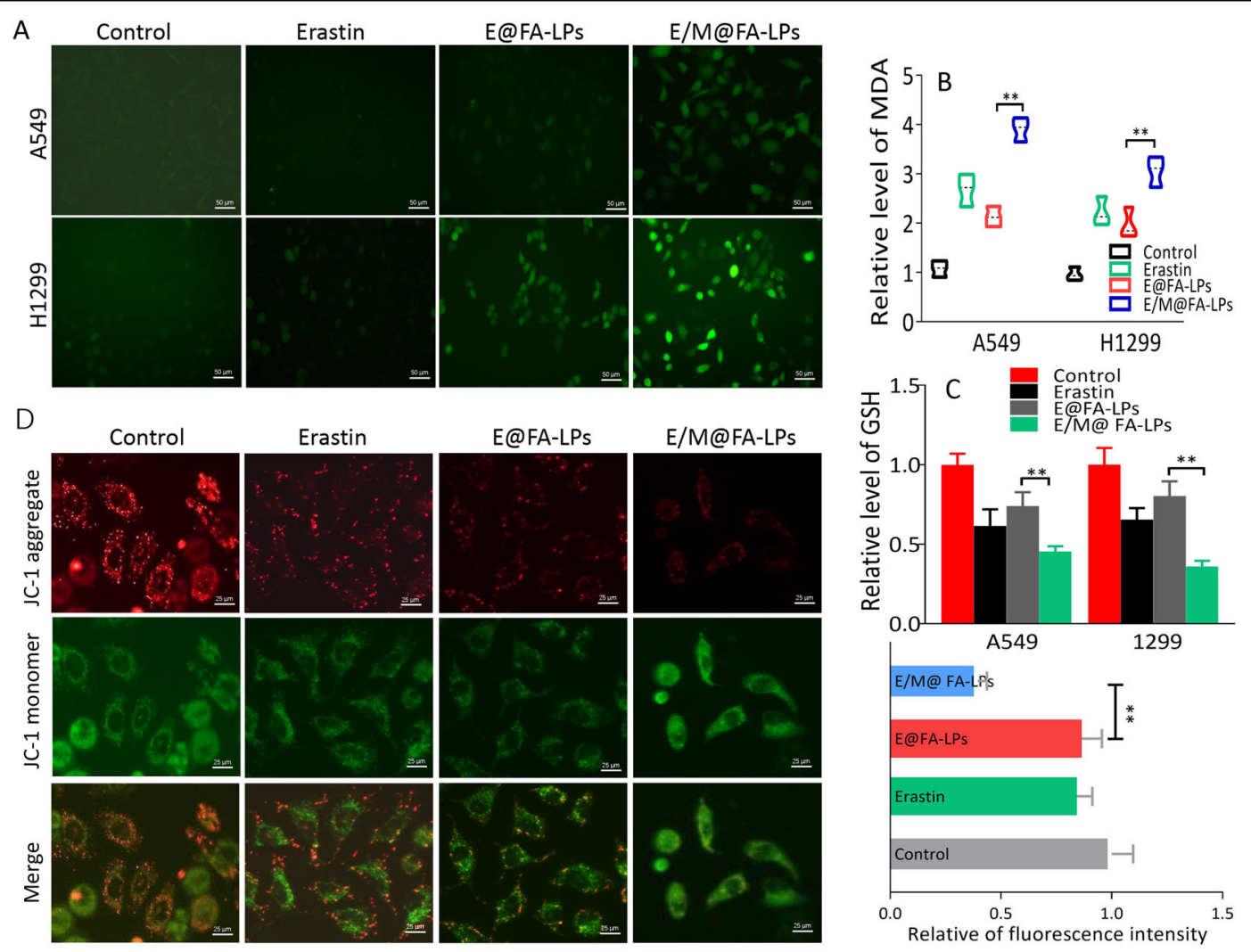

Fig. 6 E/M@FA-LPs enhanced oxidative stress. a Overgeneration of ROS treated with E/M@FA-LPs in A549 and H1299 cells. Scale bar represents $50 \mu \mathrm{m}$. b MDA content in different groups of A549 and H1299 cells, E/M@FA-LPs group had the highest MDA overproduction. c Intracellular GSH content in different groups of A549 and H1299 cells; A549 and H1299 cells treated with E/M@FA-LPs show the most obvious GSH decline. d Mitochondrial membrane potential was detected by JC-1. Scale bar represents $25 \mu \mathrm{m}$. All experiments were repeated at least three times and representative data are shown. Data are means $\pm \mathrm{SEM} ;{ }^{* *} p<0.01$.

uncover the mechanism whereby $M T 1 D P$ acts in the cell defense from oxidative stress, we explored the relationship between MT1DP and NRF2 in NSCLC samples. We found a negative correlation between the MT1DP and NRF2. Exogenous MT1DP inhibited NRF2 in A549 and H1299 cells; however, ectopic expression of NRF2 had less effect on regulating MT1DP. Overall, these results suggested that NRF2 acts downstream of MT1DP.

The interaction between IncRNAs and miRNA contributes to the occurrence of various diseases ${ }^{22,23}$; some lncRNAs work as competing endogenous RNAs to regulate target genes ${ }^{24,25}$. In this study, we found that exogenous MT1DP downregulated intracellular miR-365a$3 p$. The results of an RNA pulldown assay showed that MT1DP directly bound miR-365a-3p. Besides, a dualluciferase assay showed that miR-365a-3p targeted NRF2 mRNA. Moreover, the MT1DP-mediated inhibition of NRF2 was rescued by a miR-365a-3p inhibitor, hinting that the regulation of NRF2 by MT1DP was miR-365a-3p dependent.

In this work, we further developed FA-LPs to improve the bioavailability of erastin and its therapeutic efficacy towards ferroptosis-insensitive NCSLC cancer by thinfilm evaporation technique. E/M@FA-LPs has shown a uniform particle size of $174 \mathrm{~nm}$ and a narrow range of distribution, which achieved superior tumor accumulation because of the favorable enhanced permeability and retention effect ${ }^{26}$. E/M@FA-LPs have presented an increased antitumoral efficacy, compared with erastin alone or E@FA-LPs, restraining cell proliferation, migration, and invasion. We found that E/M@FA-LPs promoted ferroptosis of NSCLC cells with excessive amounts of ferrous ion. Treatment with E/M@FA-LPs resulted in increased mitochondrial membrane density and shrunken mitochondria with decreased $\Delta \psi \mathrm{M}$. We discovered that E/M@FA-LPs combined with erastin depressed cell viability and promoted ferroptosis of NSCLC cells; E/ M@FA-LPs impaired cell ability to cope with oxidative stress in A549 and H1299 cells, as shown by overproduction of ROS.

The role of E/M@FA-LPs-induced ferroptosis was also investigated in vivo. We found that E/M@FA-LPs reduced xenograft tumor volume and weight compared to erastin, which was accompanied by overgeneration of lipid 

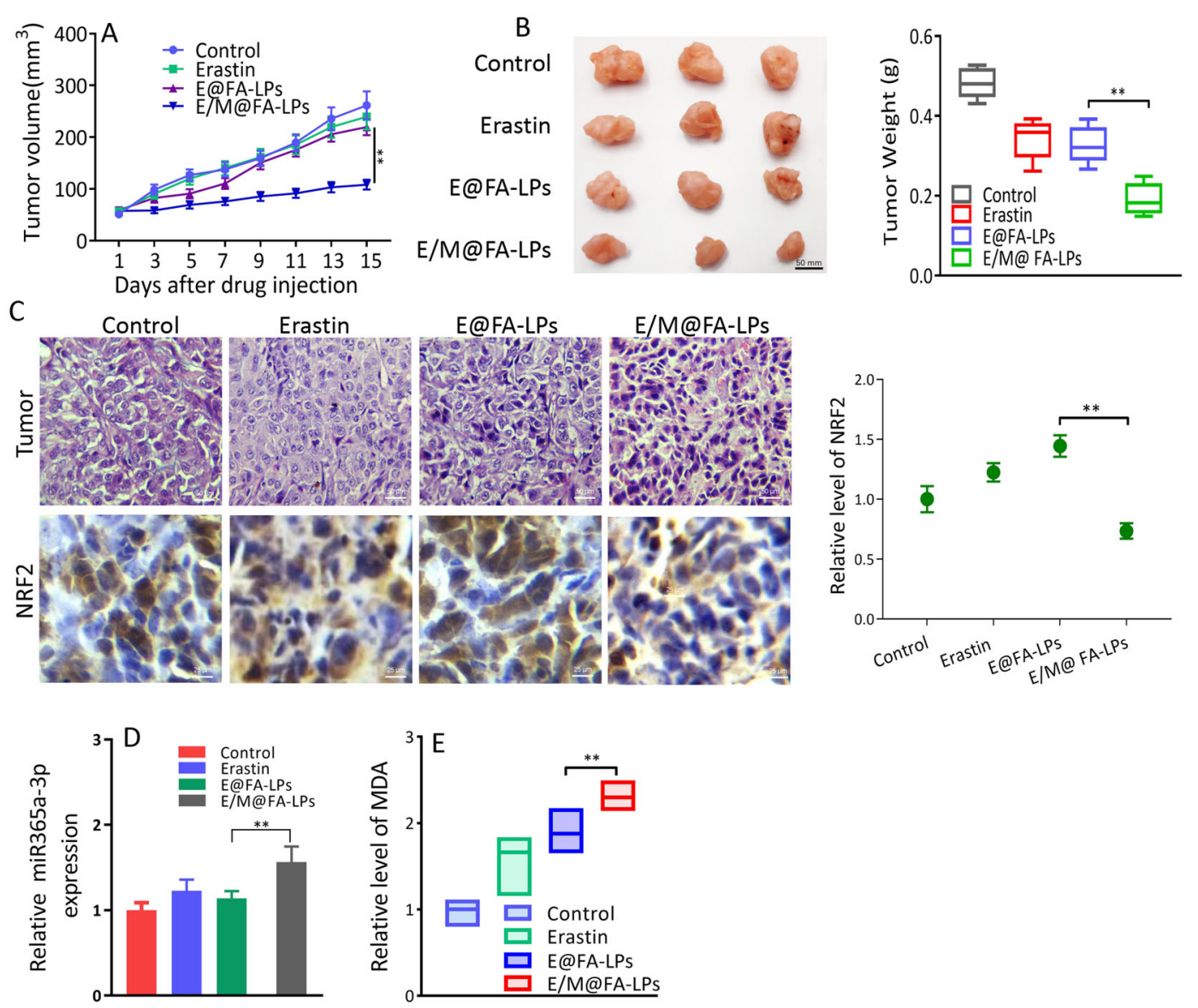

Fig. 7 Anti-tumor effects of E/M@FA-LPs in vivo. a Powerful growth inhibition of E/M@FA-LPs on subcutaneous xenografts. $\mathbf{b}$ Weight of xenograft tumors; the weight of xenograft tumors treated with E/M@FA-LPs was lighter. $\mathbf{c}$ H\&E-stained images and immunohistochemistry analysis of NRF2 in xenograft tumors. $\mathbf{d}$ miR-365a-3p expression in xenograft tumors; miR-365a-3p expression was raised in E/M@FA-LPs group. e Overproduction of MDA in xenograft tumors treated with E/M@FA-LPs. All experiments were repeated at least three times and representative data are shown. Data are means $\pm \mathrm{SEM} ;{ }^{* *} p<0.01$.

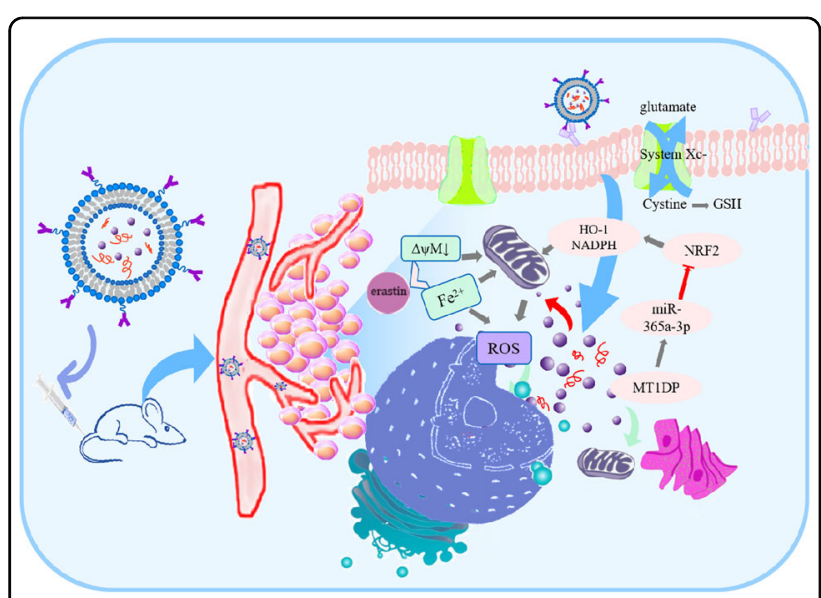

Fig. 8 Mechanism of E/M@FA-LPs antitumor in NSCLC xenograft model in mice. Treatment with E/M@FA-LPs led to overgeneration of ROS, ferrous iron and GSH depletion-induced ferroptosis, and depressed growth of xenograft tumors via modulating miR-365a-3p/NRF2 axis. peroxides. E/M@FA-LP-treated xenograft tumors showed lower expression of NRF2 and elevated miR-365a-3p levels (Fig. 8). In conclusion, we propose that MT1DP sensitized NSCLC cells to erastin-induced ferroptosis by regulating the miR-365a-3p/NRF2 signaling pathway, and in particular that E/M@FA-LPs might be considered as a successful strategy to sensitize tumors to ferroptosis.

\section{Acknowledgements}

This work was supported by grants from the National Natural Science Foundation of China (81472365, 81774125, and 81802474).

\section{Author details}

'Department of Pathology, Weifang Medical University, Weifang, Shandong Province 261014, China. ${ }^{2}$ The Second Department of Health Care, Weifang People's Hospital, Weifang, Shandong Province 261041, China. ${ }^{3}$ Department of Pharmacology, Weifang Medical University, Weifang, Shandong Province 261014, China

\section{Conflict of interest}

The authors declare that they have no competing interests. 


\section{Publisher's note}

Springer Nature remains neutral with regard to jurisdictional claims in published maps and institutional affiliations.

Supplementary Information accompanies this paper at (https://doi.org/ 10.1038/s41419-020-02939-3).

Received: 18 February 2020 Revised: 10 August 2020 Accepted: 26 August 2020

Published online: 14 September 2020

\section{References}

1. Stockwell, B. R. et al. Ferroptosis: a regulated cell death nexus linking metabolism, redox biolofy and disease. Cell 171, 273-285 (2017).

2. Dixon, S. J. et al. Ferroptosis: an iron-dependent form of nonapoptotic cell death. Cell 149, 1060-1072 (2012).

3. Conrad, M. et al. Regulation of lipid peroxidation and ferroptosis in diverse species. Genes Dev. 32, 602-619 (2018).

4. Yang, W. S. et al. Regulation of ferroptotic cancer cell death by GPX4. Cell 156, 317-331 (2014).

5. $\mathrm{Yu}, \mathrm{Y}$. et al. The ferroptosis inducer erastin enhances sensitivity of acute myeloid leukemia cells to chemotherapeutic agents. Mol. Cell. Oncol. 2, e1054549 (2015).

6. Suzuki, T. \& Yamamoto, M. Molecular basis of the Keap1-Nrf2 system. Free Radic. Biol. Med. 88, 93-100 (2015)

7. Lignitto, L. et al. Nrf2 activation promotes lung cancer metastasis by inhibiting the degradation of Bach1. Cell 178, 316-329 e318 (2019).

8. Sun, X. et al. Activation of the p62-Keap1-NRF2 pathway protects against ferroptosis in hepatocellular carcinoma cells. Hepatology 63, 173-184 (2016).

9. Dodson, M., Castro-Portuguez, R. \& Zhang, D. D. NRF2 plays a critical role in mitigating lipid peroxidation and ferroptosis. Redox Biol. 23, 101107 (2019).

10. Valadkhan, S. \& Valencia-Hipolito, A. IncRNAs in stress response. Curr. Top. Microbiol. Immunol. 394, 203-236 (2016).

11. Fuschi, P., Maimone, B., Gaetano, C. \& Martelli, F. Noncoding RNAs in the vascular system response to oxidative stress. Antioxid. Redox Signal. 30, 992-1010 (2019).

12. Gao, M. et al. Long non-coding RNA MT1DP shunts the cellular defense to cytotoxicity through crosstalk with $\mathrm{MT1H}$ and RhoC in cadmium stress. Cell Discov. 4, 5 (2018).
13. Gao, M. et al. LncRNA MT1DP aggravates cadmium-induced oxidative stress by repressing the function of $\mathrm{Nrf} 2$ and is dependent on interaction with miR365. Adv. Sci. 5, 1800087 (2018).

14. $\mathrm{Yu}, \mathrm{H}$. et al. Paclitaxel-loaded core-shell magnetic nanoparticles and cold atmospheric plasma inhibit non-small cell lung cancer. Growth ACS Appl. Mater. Interfaces 10, 43462-43471 (2018).

15. Gao, Q. et al. Long non-coding RNA Gm2199 rescues liver injury and promotes hepatocyte proliferation through the upregulation of ERK1/2. Cell Death Dis. 9, 602 (2018)

16. Furukawa, M. \& Xiong, Y. BTB protein Keap1 targets antioxidant transcription factor Nrf2 for ubiquitination by the Cullin 3-Roc1 ligase. Mol. Cell. Biol. 25 162-171 (2005).

17. Gai, C. et al. Acetaminophen sensitizing erastin-induced ferroptosis via modulation of Nif2/heme oxygenase-1 signaling pathway in non-small-cell lung cancer. J. Cell. Physiol. 235, 3329-3339 (2020).

18. Fan, Z. et al. Nrf2-Keap1 pathway promotes cell proliferation and diminishes ferroptosis. 6, e371, https://doi.org/10.1038/oncsis.2017.65 (2017).

19. Huang, $\mathrm{H}$. et al. Downregulation of Keap1 contributes to poor prognosis and Axitinib resistance of renal cell carcinoma via upregulation of $\mathrm{Nrf2}$ expression. Int. J. Mol. Med. 43, 2044-2054 (2019).

20. Houessinon, A. et al. Metallothionein-1 as a biomarker of altered redox metabolism in hepatocellular carcinoma cells exposed to sorafenib. Mol. Cancer 15, 38 (2016).

21. Liang, G. Y. et al. Expression of metallothionein and Nrf2 pathway genes in lung cancer and cancer-surrounding tissues. World J. Surg. Oncol. 11, 199 (2013).

22. Wang, W. et al. Long non-coding RNA UCA1 promotes malignant phenotypes of renal cancer cells by modulating the miR-182-5p/DLL4 axis as a ceRNA. Mol. Cancer 19, 18 (2020).

23. Cremer, S. et al. Hematopoietic deficiency of the long noncoding RNA MALAT1 promotes atherosclerosis and plaque inflammation. Circulation 139 1320-1334 (2019).

24. Wang, $Y$. et al. Long noncoding RNA DANCR, working as a competitive endogenous RNA, promotes ROCK1-mediated proliferation and metastasis via decoying of miR-335-5p and miR-1972 in osteosarcoma. Mol. Cancer 17, 89 (2018).

25. Zhu, K. P. et al. Analyzing the interactions of mRNAs and ncRNAs to predict competing endogenous RNA networks in osteosarcoma chemo-resistance. Mol. Ther. 27, 518-530 (2019).

26. Maeda, H., Nakamura, H. \& Fang, J. The EPR effect for macromolecular drug delivery to solid tumors: improvement of tumor uptake, lowering of systemic toxicity, and distinct tumor imaging in vivo. Adv. Drug Deliv. Rev. 65, 71-79 (2013). 